\title{
Implementation of High Magnetization System for Performance Enhancement of Magnetic Flux Leakage Tool
}

\author{
Sung-Ho Cho ${ }^{1,4 *}$, Hui-Ryong Yoo ${ }^{1}$, Dong-Ku Kim ${ }^{1}$, Dae-Jin Park ${ }^{1}$, Yong-Woo Rho ${ }^{1}$, \\ Kang Seo ${ }^{2}$, Gwan Soo Park ${ }^{2}$, Doo-Hyun Choi ${ }^{3}$, and Sung-Jin Song ${ }^{4}$ \\ ${ }^{1} R \& D$ Division, Korea Gas Corporation, Incheon, Korea \\ ${ }^{2}$ School of Electrical Engineering, Pusan National University, Busan, Korea \\ ${ }^{3}$ School of Electronics Engineering, Kyungpook National University, Daegu, Korea \\ ${ }^{4}$ School of Mechanical Engineering, Sungkyunkwan University, Kyounggi-Do, Korea
}

(Received 23 August 2010, Received in final form 8 October 2010, Accepted 8 October 2010)

\begin{abstract}
This paper discusses the effectiveness of high magnetization saturation in ILI (In-Line Inspection) using an MFL (Magnetic Flux Leakage) tool, and introduces a practical method for improving the magnetization level together with the piggability. Thin steel plates, replacing the conventional wire brushes were used as conductors to transfer the magnetic flux to the pipe wall. The newly designed MFL tool was compared with the conventional version by means of FEM (Finite Element Method) analysis and full-scale experiments. In the results, the newly developed magnetization system obtained a stronger MFL signal amplitude, specially 2.7 times stronger, than that obtained by the conventional magnetization system for the same defect dimensions.
\end{abstract}

Keywords : defects, magnetic flux leakage tool, magnetization system, piggability, pipeline inspection, NDE

\section{Introduction}

ILI (In-Line Inspection) with either MFL (Magnetic Flux Leakage) tools or ultrasonic tools is the best current technology for inspection of the integrity of buried pipelines. In the case of gas pipelines, field operators usually choose MFL tools, because ultrasonic tools require a couplant for wave propagation [1,2]. Two performance factors make MFL tools perferable: piggability, and data analysis accuracy.

The piggability is the capability of an inspection tool to pass through pipe with tight bends or pipe with internal diameter reductions. MFL tools recently have been passed through particularly tight bends radii of which were more than 1.5 times smaller than the outside diameter of the pipelines. And as regards MFL data analysis performance, it can be measured according to the accuracy of defect characterization and the POD (Probability Of Detection). These can be improved greatly with a strong magnetization system, because such a system can generate stable, reliable and robust MFL signals against velocity and remanent

*Corresponding author: Tel: +82-32-810-0343

Fax: +82-32-810-0339, e-mail: shcho@kogas.or.kr magnetization effects [3].

Not surprisingly, development of a new-concept MFL tool having both good piggability and a strong magnetization system has been a high priority among pipeline researchers. Unfortunately, it is very difficult to implement both at the same time, as the trade-off relationship between them usually means that improvement of one results in the diminishment of the other.

In this paper, we will discuss the design concept of a new MFL tool that has been under development at Korea Gas Corporation since 2004.

First, many thin steel plates were applied instead of wire brushes, in order to impart as strong a magnetic flux to the pipe wall as possible. The thin steel plates, moreover, allowed for removal of the support wheels, as they can bear the more than 1.4 ton weight of the tool body and its elastic force while maintaining the tool in the center of a pipeline. Second, to increase the magnetic power, permanent magnets were arranged circularly on an unmovable polygon-shaped back-yoke, replacing the conventional tool's movable back-yoke with spring. Significantly, this combination of thin steel plates and the circular permanent magnet arrangement not only increases the magnetic strength, and renders it uniform and consistent, but also 
enhances the piggability by allowing for removal of the support wheels and, thereby, shortening the tool body.

In the present study, the new MFL tool was designed and fabricated as just explained. Through an FEM (Finite Element Method) analysis, the magnetic field strength of the new tool was compared with that of the conventional tool, which, as already stated, employs the movable magnetization system, wire brushes and support wheels. Also, full-scale comparative experiments were conducted to evaluate the overall performance of the new tool.

\section{Design and Fabrication of New Magnetization System}

When a magnetization system is placed inside a pipe wall, most of the flux lines pass through that wall. That is, the pipe wall is the preferred path for the flux. With most flux lines concentrated in the pipe wall, local wearing of pipe wall thickness in a metal loss region leads to flux leakage. In such a metal loss region, a sensor array positioned on the inside of the pipe measures the magnetic field adjacent to the pipe wall, and a hall sensor senses higher density than that of the nominal pipe area. In this manner, an MFL tool detects anomalies that cause magnetic flux leaks.

Fig. 1 shows a typical conventional tool design favored by many companies. Usually the design consists of a magnetization system, a sensor system, support wheels, a data acquisition system and other components.

Among the common features of the conventional tool, wire brushes are used as a magnetic conductor to apply a magnetic flux to a pipe wall, while the back-yoke made of low carbon steel, completes a magnetic flux circuit with the wall. There are two types of back-yoke. One is movable back-yoke that can move for center of tool as same as

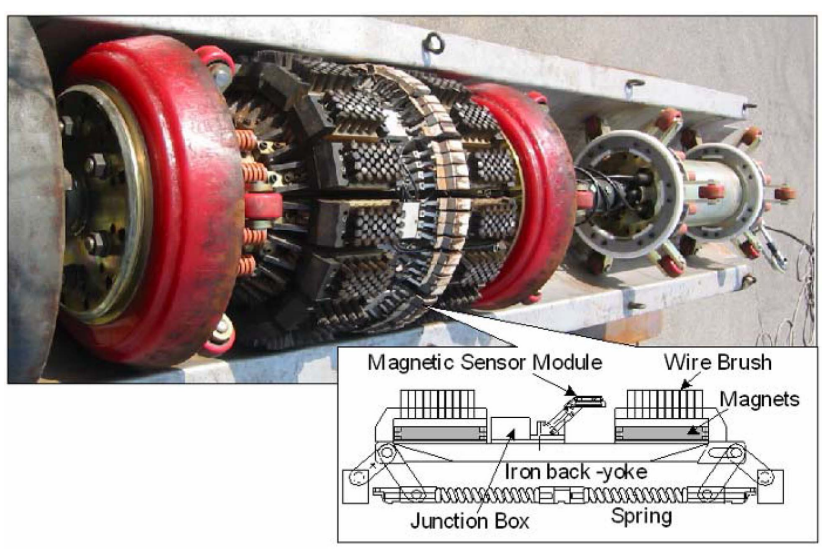

Fig. 1. Typical design of conventional MFL Tool. stroke of spring, and so it offers the advantage of exact estimation of the tool diameter when it compressed. The other is an unmovable back-yoke, in which case the length of the brush must get longer than that of movable backyoke considering the piggability. Therefore, it is important to magnetize a pipe wall uniformly in the circumferential direction in order to obtain reliable signals for defect areas. But the weight of a tool more than 1.4 tons might interfere the uniform magnetization of a whole pipe wall. Therefore, most MFL tools incorporate support wheels to maintain themselves in the centers of a pipes.

In the present study, in order to enhance magnetization capability of an MFL tool, minimize its length and help to maintain it in the center of a pipe without support wheels, a new MFL tool was designed and fabricated. Fig. 2 shows the newly developed tool and three-dimensional (3D) schematics of its magnetization system.

As Fig. 2 makes apparent, many thin steel plates were applied in place of the usual wire brushes to transfer the magnetic flux to the pipe wall as effectively as possible. These thin steel plates can bear the weight of the tool body with its elastic force, allowing a designer to remove the support wheels from the tool body. The spaces, where the support wheels had been, therefore, could be used to minimize the MFL tool length and maximize the length of the magnetization system. Maximization of the length of magnetization system is essential enhancing the magnetic power and generating strong, uniform, and consistent magnetic flux leakage signals thereby.

Whereas, in the conventional design shown in Fig. 1, magnets are affixed to the movable flat back-yoke, in the new design, permanent magnets are arranged circularly on an unmovable back-yoke manufactured in the shape of a polygon. The circular arrangement of the magnets increases the transmission efficiency of the magnetic flux in the magnetic circuit consisting of magnets, brushes, backyoke and the pipe wall.

A combination of thin steel plates and circular arrangement of permanent magnets not only improves the magnetic flux density but also enhances piggability.

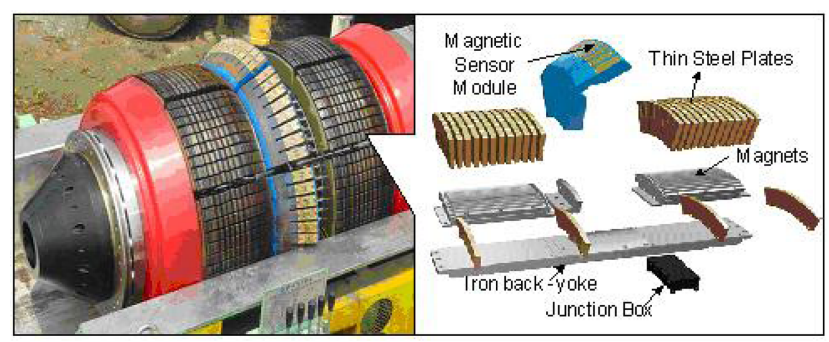

Fig. 2. New 3D design of magnetization system. 


\section{Finite Element Method (FEM) Analysis}

\subsection{Magnetic Field Analysis of Magnetization System}

The magnetic field in the magnetic flux leakage system can be described by the Maxwell equations [4];

$$
\begin{aligned}
& \nabla \times H=J \\
& B=\mu_{0}\left(H+M^{\prime}\right) \\
& B=\nabla \times A
\end{aligned}
$$

Where $B$ is the magnetic flux density, $H$ is the magnetic field strength, $J$ is the current density, $A$ is the magnetic vector potential and $\mu_{0}$ is the magnetic permeability of the vacuum. In the present study, the main source of the magnetic field of the sensing system was a permanent magnet and so the magnetization $M^{\prime}$ was represented by the sum of the reversible $(\chi H)$ and irreversible $(M)$ component as in Eq. (4):

$$
M^{\prime}=\chi H+M
$$

The magnetic field in this case is represented as

$$
H=v B-v_{r} M
$$

where $v=1 / \mu, \mu=\mu_{0} \mu_{r}, v_{r}=1 / \mu_{r}, \mu_{r}=1+\chi$.

Substituting these equations into (1) and (3), the Coulomb gauge and vector relation yields

$$
-(\nabla \cdot v \nabla) A=J+v_{r} \nabla \times M
$$

In finite element analysis, the analysis area is divided into finite elements and fixed or free boundaries. A weighting function $\mathrm{W}$ satisfying the fixed boundary conditions is defined. Multiplying this weighting function by (6) and integrating it into all analysis domains affords

$$
\int_{\Omega}\left\{-v \nabla W \nabla \cdot A+W J-v_{r} \nabla W \times M\right\} d \Omega=0
$$

To apply the energy minima criteria, the magnetic vector potential can be calculated.

\subsection{Design of High Magnetic Field System}

Sensing signals in an MFL system can be affected by several inevitable disturbances such as variations in the localized stress of the pipe wall, fluctuations of the tool speed (eddy current effects), changes in the pipe wall material and buildup of debris on the magnetic circuit itself. These, if the sensitivities of an MFL system are to be enhanced, need to be eliminated. In the present research, we increased the number of operating points on the magnetization curve so that the sensing signals depended more on the metal loss of the pipeline.

The basic MFL principle requires magnetic saturation of the pipe wall. If an MFL tool is functioning a low magnetization area, it is difficult to avoid the adverse effects of magnetic disturbance factors and the resultant measurement errors, simply because magnetic disturbance factors occur in such areas.

Fig. 3 illustrates the incremental permeability which is defined by Eq. (8):

$$
\mu_{\text {incremental }}=\frac{A B}{\Delta H}
$$

In the figure, the incremental permeability of OP2 in the high-field-strength region is smaller than that of OP1 in low-field-strength region, ands so we can reduce the magnetic field variations caused by inevitable disturbance factors such as debris, localized stress, and speed fluctuations. In the new design, we set the operating point in the high magnetic field region so as to reduce unwanted disturbances and to increase the sensibilities of the magnetic sensors.

It is reasonable to expect that use of a high magnetization MFL tool will result in improvements to the output signal such as increases of the signal amplitude and noise reductions, and that the signal will be sensitive only to wall thickness changes or metal loss defects.

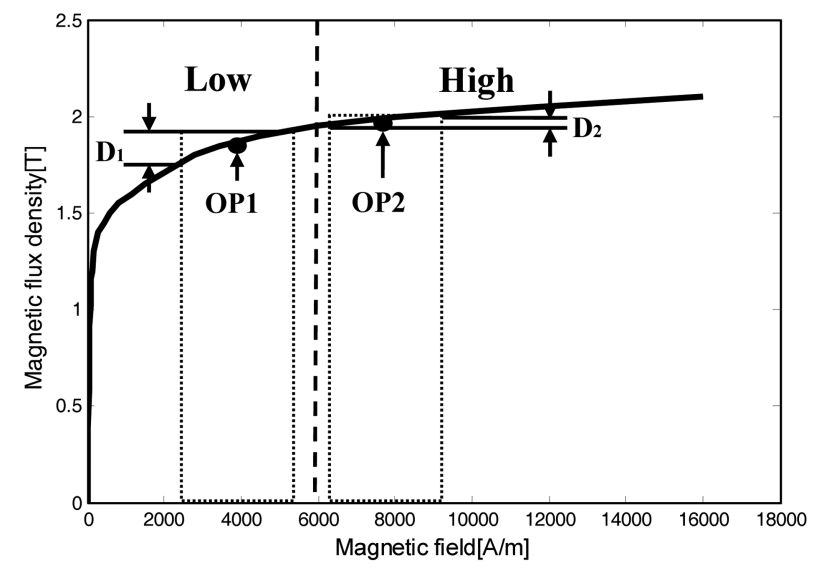

Fig. 3. Operating Points on B-H curve.
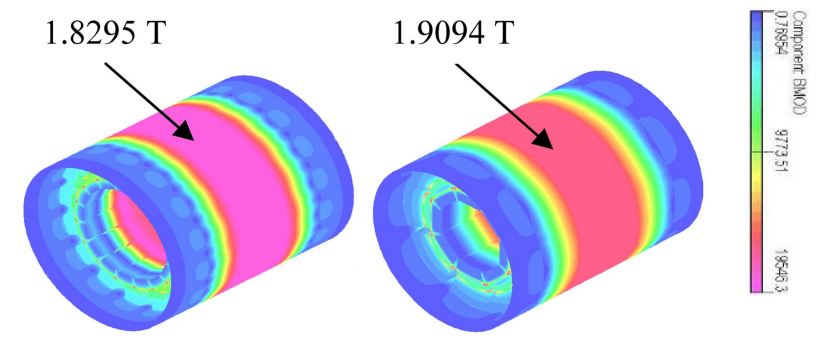

(a) Conventional magnetization system

(b) New magnetization system

Fig. 4. Magnetic fields distributions of conventional and new magnetization systems. 


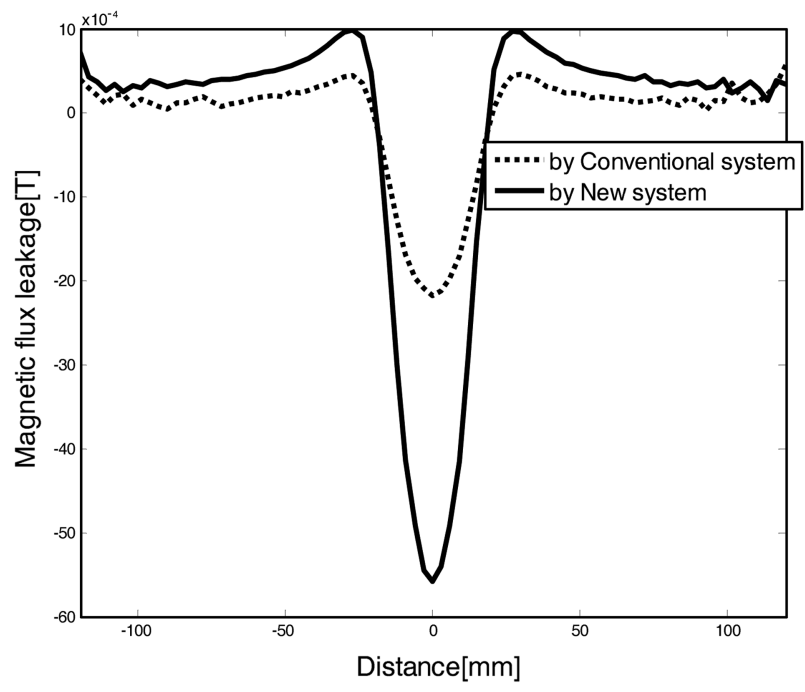

Fig. 5. Simulated MFL axial signal defect (length: $35 \mathrm{~mm}$, width: $35 \mathrm{~mm}$, depth: $5.25 \mathrm{~mm}$ ).

In these magnetization systems, the thickness of the magnet is thin and the polarity of the magnet is nonsymmetrical, so 3D analysis is essential. The number of nodes and elements are approximately 40,000 and 200,000, respectively.

Fig. 4 shows the magnetic fields distributions of the conventional and new magnetization systems. The magnetization level of the new system was increased 9.3\% over that of the conventional one.

To determine the extent of the sensing signal increase, a pipe wall with metal loss was analyzed. Fig. 5 shows the results of a 3D FEM analysis at the defect $(2 \mathrm{t} \times 2 \mathrm{t} \times 0.3 \mathrm{t}$, where $t=17.5 \mathrm{~mm}$ ) for magnetization by the conventional and new MFL tools. As expected, the amplitude of the magnetic flux leakage with the new magnetization system increased 2.5 times over that with conventional system.

\section{Experiments and Results}

In order to verify the performance of the developed MFL tool, full-scale experiments were undertaken at KPSF (KOGAS Pipeline Simulation Facility). KPSF consists of $300 \mathrm{~m}$ of 30 inch diameter pipeline including tees, offtakes, valves, bends, casing and a huge number of artificial defects to simulate realistic ILI of a pipeline.

The performance was evaluated by comparing the amplitude of the MFL signals generated by the new and conventional tools at the same defect.

Fig. 6 plots the measured MFL axial signals by the conventional and new MFL tools at a defect of $2 t$ (length) $\times 2 \mathrm{t}($ width $) \times 0.3 \mathrm{t}($ depth $)$, where $\mathrm{t}=17.5 \mathrm{~mm}$. The MFL

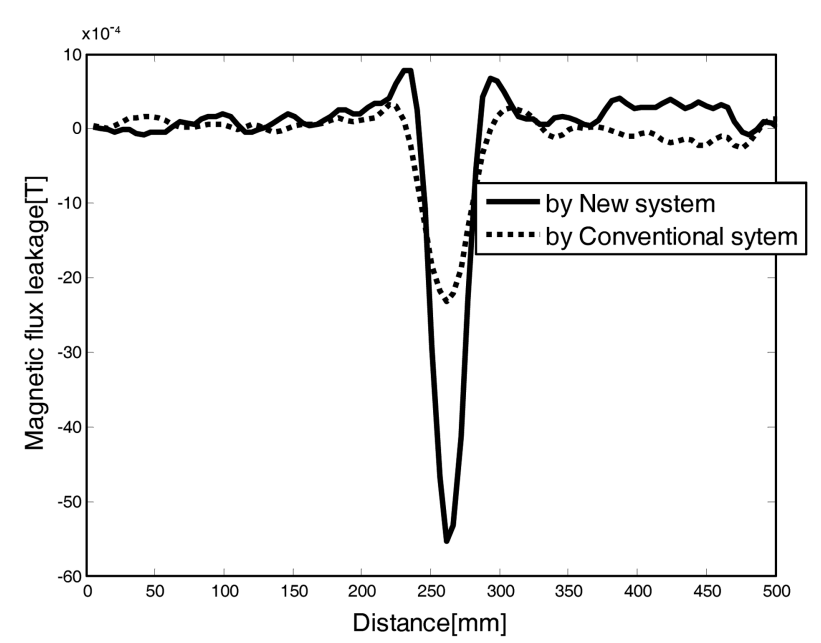

Fig. 6. Measured MFL axial signal. (defect length: $35 \mathrm{~mm}$, width: $35 \mathrm{~mm}$, depth: $5.25 \mathrm{~mm}$ ). The dotted line is signal measured by the conventional MFL tool; the solid line is the signal measured by the newly developed MFL tool.

Table 1. Piggability Comparison.

\begin{tabular}{lcc}
\hline \hline \multicolumn{1}{c}{ Items } & $\begin{array}{c}\text { Newly Developed } \\
\text { tool }\end{array}$ & Conventional tool \\
\hline Minimum Continuous & $660 \mathrm{~mm}$ & $703 \mathrm{~mm}$ \\
$\quad$ Bore & $642 \mathrm{~mm}$ & $668 \mathrm{~mm}$ \\
Minimum Local Bore & $1180 \mathrm{~mm}$ & $1733 \mathrm{~mm}$ \\
Length of Magnetization & & $3 \mathrm{D}$ \\
$\quad$ System & $1.5 \mathrm{D}$ & Minimum Bend Radius \\
& (D: Diameter of Pipe) (D: Diameter of Pipe) \\
\hline
\end{tabular}

axial signal by the new MFL tool was about 2.7 times stronger than that by the conventional MFL tool. Additionally, Fig. 7 shows the amplitudes of the MFL axial signals at various defects of various sizes. These results, prove that the performance of the new MFL tool is much superior to that of the conventional tool.

Table 1 shows a piggabilty parameter comparison. The results clearly indicate that with the shortened length of the newly developed tool, the piggability was considerably improved.

\section{Conclusion}

In this paper, we have discussed the effectiveness of high magnetization saturation in the use of MFL tools, and we proposed a practical and effective method to improve both the magnetization level and piggability of tools using a combination of thin steel plates and circularly arranged permanent magnets. After the new MFL tool 


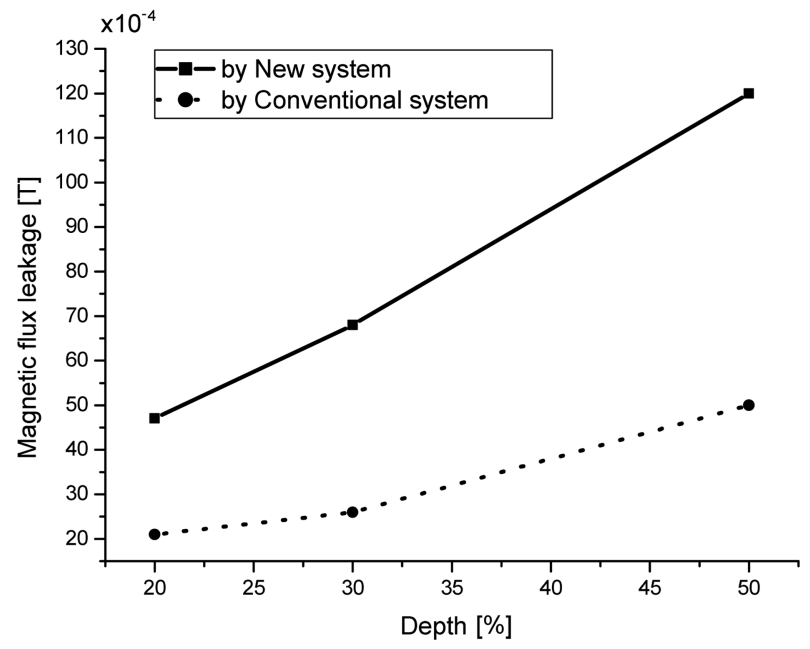

(a) Defect size : $2 \mathrm{t} \times 2 \mathrm{t}$

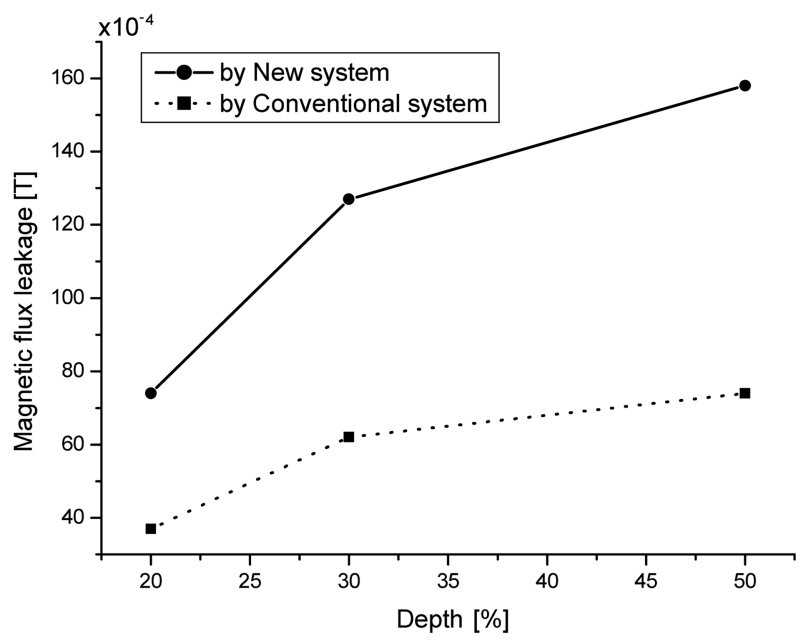

(b) Defect size : $4 \mathrm{t} \times 4 \mathrm{t}$

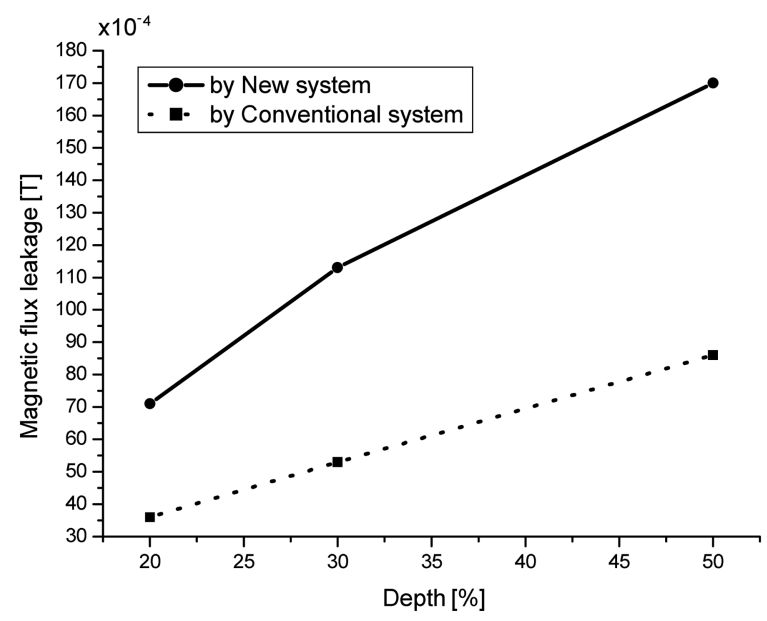

(c) Defect size : $6 \mathrm{t} \times 6 \mathrm{t}$

Fig. 7. Comparison of amplitudes of MFL axial signal by conventional and new MFL tool (thickness of pipeline $\mathrm{t}=17.5 \mathrm{~mm}$ ).

was manufactured, an FEM analysis and full-scale experiments were conducted at the KPSF, proving that the proposed design was very effective in improving the tool's detection of various defects. In fact, the tool, having been verified in its performance, has been used in real KOGAS pipeline since 2007.

\section{References}

[1] Stephen Westwood and Sharon Cholowsky, Independent experimental verification of the sizing accuracy of mag- netic flux leakage tools, 7th International pipeline conference (2003).

[2] John Tiratsoo, Pipeline Pigging and Integrity Technology, Clarion, Portsmouth (2003) pp. 307-311.

[3] T. Bubenik, J. Nestleroth, R. Eiber, and B. Saffell, Magnetic Flux Leakage Technology for Natural Gas Pipeline Inspection, Gas Research Institute Topical Report GRI 910367 (1992).

[4] D. L. Atherton and M. G. Daly, Finite Element Calculation of Magnetic Leakage Detector Signals, NDT International 20, 235 (1987). 